\title{
Effects of Carvedilol in Heart Failure due to Dilated Cardiomyopathy. Results of a Double-blind Randomized Placebo-controlled Study (CARIBE Study)
}

\author{
Paulo Roberto Chizzola, Humberto Felício Gonçalves Freitas, Márcia Azevedo Caldas, \\ Joicely Melo da Costa, Cláudio Meneghetti, Norma Vasconcelos Saldanha Marinho, \\ Alfredo José Mansur, José Antonio Franchini Ramires, Edimar Alcides Bocchi
}

São Paulo, SP

Objective - To assess the effects of carvedilol in patients with idiopathic dilated cardiomyopathy.

Methods - In a double-blind randomized placebocontrolled study, 30 patients ( 7 women) with functional class II and III heart failure were assessed. Their ages ranged from 28 to 66 years (mean of $43 \pm 9$ years), and their left ventricular ejection fraction varied from $8 \%$ to $35 \%$. Carvedilol was added to the usual therapy of 20 patients; placebo was added to the usual therapy of 10 patients. The initial dose of carvedilol was $12.5 \mathrm{mg}$, which was increased weekly until it reached $75 \mathrm{mg} /$ day, according to the patient's tolerance. Clinical assessment, electrocardiogram, echocardiogram, and radionuclide ventriculography were performed in the pretreatment phase, being repeated after 2 and 6 months of medication use.

Results $-A$ reduction in heart rate $(p=0.016)$ as well as an increase in left ventricular shortening fraction $(p=0.02)$ and in left ventricular ejection fraction $(p=0.017)$ occurred in the group using carvedilol as compared with that using placebo.

Conclusion - Carvedilol added to the usual therapy for heart failure resulted in better heart function.

Key words: heart failure, beta-blocker, dilated cardiomyopathy

Instituto do Coração do Hospital das Clínicas - FMUSP Mailing address: Paulo Roberto Chizzola - Incor - Divisão de Clínicas Av. Dr. Enéas C. Aguiar, 44 - 05403-000 - São Paulo, SP, Brazil
The objective of treatment in patients with heart failure is to improve symptoms, delay the evolution of the ventricular dysfunction, and reduce mortality.

Usually, the medicamentous therapy is based on the association of diuretics, digitalis, ACE inhibitors or nitrates, and vasodilators ${ }^{1}$. Of these drugs, only the ACE inhibitors reduce mortality ${ }^{2-4}$. Despite the clear benefits of the betaadrenergic blockade in patients with ventricular dysfunction ${ }^{5}$, a reduction in the risk of death in the treatment of heart failure was only demonstrated with the use of carvedilol ${ }^{6}$, which is a nonselective third-generation beta-blocker and a vasodilator due to the alpha-1-adrenergic blockade with an antioxidant activity, and also an inhibitor of apoptosis ${ }^{7,8}$.

The present study was carried out to assess the tolerance and efficacy of the addition of carvedilol to the conventional therapy (digitalis, diuretic, ACE inhibitors or vasodilators and nitrates) of patients with severe heart failure, who, in our experience, are younger ${ }^{9}$. This conventional therapy has been described in former published studies ${ }^{10}$.

\section{Methods}

Fifty-three patients undergoing ambulatory assessment for heart transplantation selection were invited to take part in the study.

The criteria of inclusion in the study were the following: heart failure due to idiopathic dilated cardiomyopathy, functional class II or III according to the New York Heart Association classification, and the patient should be undergoing clinical follow-up and regularly using medication. The patients should also have a sinus rhythm on resting ECG, a left ventricular ejection fraction $\leq 35 \%$ in the radionuclide ventriculography, and should have a thoracic conformation allowing an appropriate echocardiographic window to obtain images to measure the diameters of the cardiac chambers.

The exclusion criteria were the following: abusive use 
of alcohol; systemic blood pressure during systole $<85 \mathrm{~mm}$ $\mathrm{Hg}$ or $>160 \mathrm{mmHg}$ and during diastole $>100 \mathrm{mmHg}$; heart rate $<60 \mathrm{bpm}$; previous use of beta-blockers for the treatment of heart failure; second degree atrioventricular block of Mobitz type II or total atrioventricular block; evidence of bronchospasm on physical examination or possibility of ongoing pregnancy.

At the beginning of the study, 53 patients were invited to take part (fig. 1). Thirteen patients refused to participate in the assessment, and 10 were excluded ( 5 had inappropriate echocardiographic windows, 2 had atrial fibrillation on electrocardiogram, one had a primary valvular heart disease, one died during the initial phase, and one became pregnant). From the 30 patients ( 7 females and 23 males), 20 were randomly distributed for the use of carvedilol and 10 for placebo. The profile of the population in the two groups in the pretreatment phase is shown in table I.

All patients were instructed about the study protocol and their participation in the study. After giving consent, each patient was randomly included in the double-blind placebo-controlled study. The study protocol was assessed and approved by the Ethics Committee of the hospital.

In the pretreatment phase, the patients underwent a clinical visit, a resting ECG, radionuclide ventriculography, an echocardiogram, measurement of serum dosage of cate-

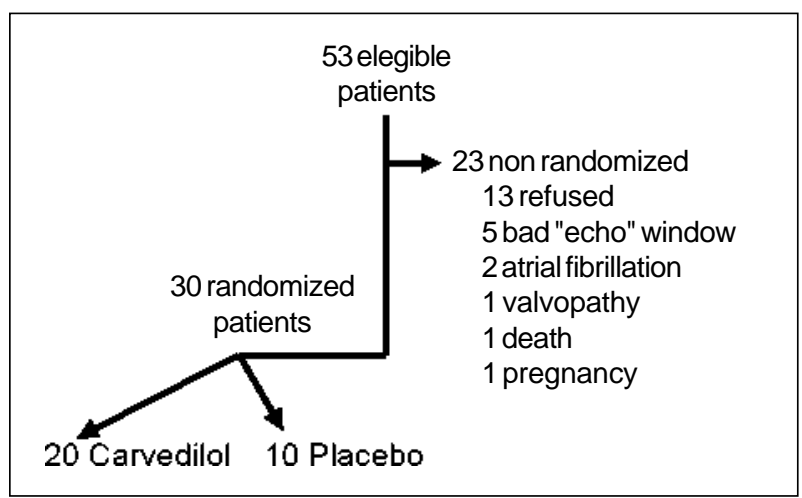

Fig. 1 - Design of the study for selecting the candidates until reaching the 30 planned patients, who were randomly distributed in the following manner: 10 patients to use placebo and 20 to use carvedilol.

Table I - Characteristics of the population in the pretreatment phase

\begin{tabular}{lcc} 
Variable & Placebo & Carvedilol \\
\hline Age (years) & $41.7 \pm 9.0$ & $45.4 \pm 9.1$ \\
Weight (kg) & $69.6 \pm 15.1$ & $65.4 \pm 11.1$ \\
Sex $(*$ male/female) & $* 5 / 2$ & $* 10 / 5$ \\
Functional class $(* \mathrm{II} / \mathrm{III})$ & $* 6 / 1$ & $* 10 / 5$ \\
LV diastolic diameter $(\mathrm{mm})$ & $77.7 \pm 14.3$ & $75.7 \pm 8.7$ \\
LV systolic diameter $(\mathrm{mm})$ & $66.4 \pm 12.7$ & $65.5 \pm 7.7$ \\
Shortening fraction $(\%)$ & $14.7 \pm 2.4$ & $13.3 \pm 1.5$ \\
LV ejection fraction $(\%)$ & $22.5 \pm 8.5$ & $21.3 \pm 6.2$ \\
Systolic blood pressure $(\mathrm{mmHg})$ & $110.3 \pm 15.1$ & $104.1 \pm 14.4$ \\
Diastolic blood pressure (mmHg) & $78.8 \pm 15.5$ & $75.3 \pm 10.2$ \\
Heart rate (bpm) & $85.7 \pm 12.7$ & $83.3 \pm 13.2$ \\
Serum noradrenaline $(\mathrm{pg} / \mathrm{ml})$ & $448 \pm 74.9$ & $550.8 \pm 341.7$ \\
& & \\
\hline
\end{tabular}

Carvedilol in heart failure due to dilated cardiomyopathy

cholamines, a hemogram, measurement of serum dosage of urea, creatinine, glucose, sodium and potassium, and an analysis of the urinary sediment.

The addition of carvedilol ( $*$ Dilatrend $^{\circledR}$ - Asta Médica) or placebo was initiated at a dosage of $6.25 \mathrm{mg}$ twice a day, increased weekly to $12.5 \mathrm{mg}$ and $25 \mathrm{mg}$ twice a day, and later to $25 \mathrm{mg}$ three times a day, according to the patient's tolerance and adherence to treatment. We tried to reach a dosage of $75 \mathrm{mg} /$ day, which is higher than the recommended $50 \mathrm{mg} /$ day ${ }^{11}$, due to the patient's clear improvement with the use of carvedilol ${ }^{12}$. After reaching the desired or tolerated maximum dosage, the patient was reassessed at a monthly medical visit or in a shorter period of time, according to clinical need. The complementary tests were repeated after 2 and 6 months of medication use.

Quantitative variables were presented in a descriptive way in tables containing the mean, the standard deviation, and the minimum and maximum values. Those variables assessed in more than one condition $\left(\Delta \mathrm{t}_{0}, \Delta \mathrm{t}_{1}\right.$ and $\left.\Delta \mathrm{t}_{2}\right)$ were studied according to the multivariate technique of profile analysis. This technique aims to simultaneously analyze the group and assessment condition variables, and their combined (interaction) and isolated effects.

The three basic hypotheses to be tested in the profile analysis are as follows: $\mathrm{H}_{01}$ : the profiles of the means of the groups studied are parallel to each other, i. e., no relation between the group factor and the assessment condition factor occurs; $\mathrm{H}_{02}$ : the profiles of the means of the studied groups coincide, i. e., no effect of the group factor occurs; $\mathrm{H}_{03}$ : the profiles of the means of the studied groups are parallel to the $\mathrm{X}$-axis, i.e., no effect of the assessment condition factor occurs. Values of $p<0.05$ were considered statistically significant ${ }^{13}$.

\section{Results}

Eight patients were excluded from the study due to the following reasons: two patients due to intolerance of the medication, one to carvedilol and the other to placebo; four patients on carvedilol due to poor adherence to the clinical follow-up; one patient using placebo suddenly died during the phase of medication dosage increase; and one patient using placebo was excluded due to a change in therapeutical regimen.

In regard to the dosage of medication of those patients who continued to be followed up, only 2 patients on carvedilol did not tolerate a dosage higher than $50 \mathrm{mg} /$ day. The remaining patients were kept on $75 \mathrm{mg} /$ day.

In regard to the evolution of the heart failure functional class (fig. 2) according to the New York Heart Association classification, among those patients using placebo, 5 remained in functional class II during the whole period of the study, one patient improved evolving from functional class III to functional class II, and another from functional class II to I. Among those patients using carvedilol, 4 remained in functional class II during the whole study, 4 patients improved, going from functional class III to II and 7 patients from functional class II to I.

* Dilatrend ${ }^{\circledR}$ - Asta Médica 


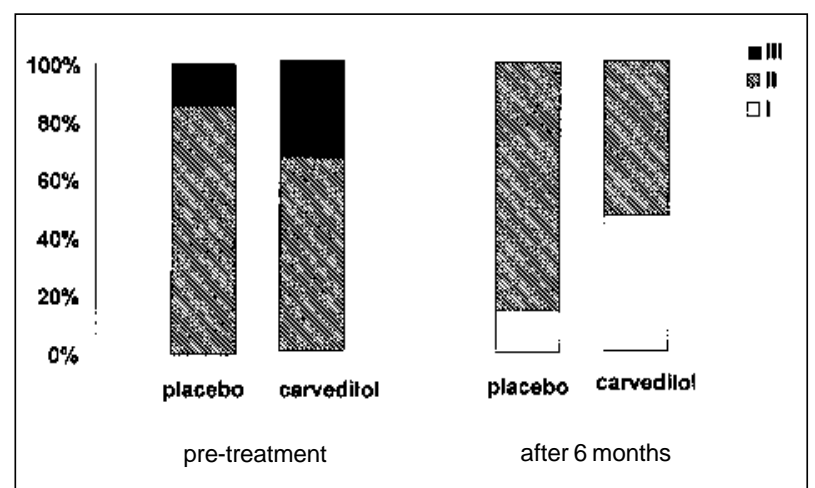

Fig. 2 - Distribution of the evolution in functional class for heart failure in the groups using placebo or carvedilol, in the pretreatment phase and after 6 months of medication use.

In regard to heart rate (fig. 3 ), a reduction in the mean occurred among those patients using carvedilol, from 83bpm to $61 \mathrm{bpm}$ after two months of drug use, and in 6 months it remained at $63 \mathrm{bpm}$. On the contrary, patients in the placebo group retained an unaltered heart rate around 80bpm during the study period $(\mathrm{p}=0.016)$.

Regarding left ventricular diameters during systole (fig. 4) and diastole (fig. 5) on echocardiogram, no significant alteration in the means of the groups during the study

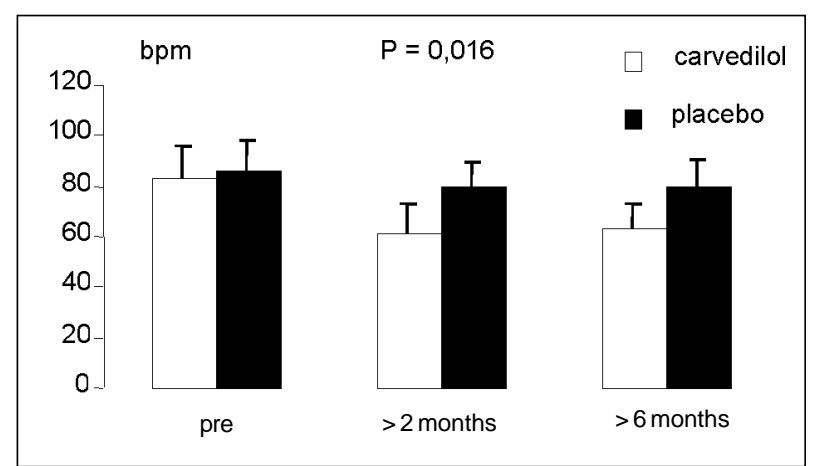

Fig. 3 - Comparison of the evolution in heart rate on resting electrocardiogram between the groups using carvedilol or placebo in the pretreatment phase (pre), after 2 months using the medication ( $>2$ months), and after 6 months using the medication (>6 months).

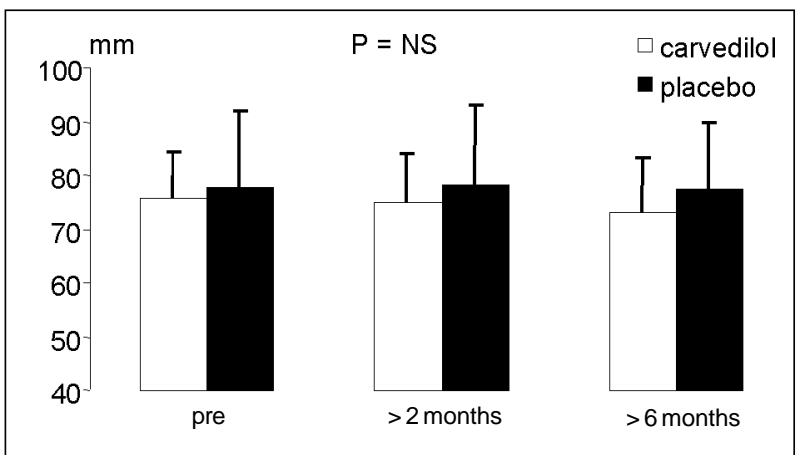

Fig. 4 - Comparison of the evolution in left ventricular diastolic diameter on echocardiogram between the groups using carvedilol or placebo in the pretreatment phase (pre), after 2 months of medication use ( $>2$ months), and after 6 months of medication use (>6 months)

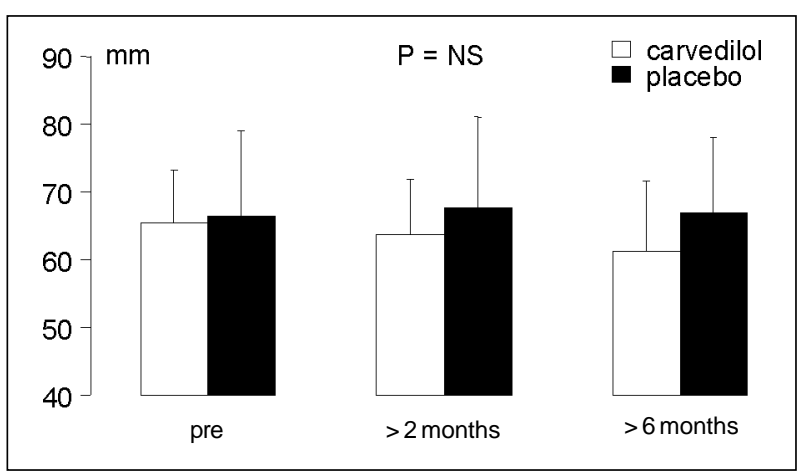

Fig. 5-Comparison of the evolution in left ventricular systolic diameter on echocardiogram between the groups using carvedilol or placebo in the pretreatment phase (pre), after 2 months of medication use ( $>2$ months), and after 6 months of medication use (>6 months).

occurred. However, an improvement in the mean of the left ventricular shortening fraction occurred (fig. 6) in the group on carvedilol, from $13.3 \%$ to $15.2 \%$ in 2 months and to $16 \%$ after 6 months of drug use. Among the patients using placebo, a drop from $14.7 \%$ to around $13.6 \%$ was observed during the study $(\mathrm{p}=0.0235)$.

In regard to left ventricular ejection fraction on radionuclide ventriculography (fig. 7), an improvement in the mean of the group on carvedilol occurred, from $21.3 \%$ to $27.3 \%$ in 2 months of drug use and $28.3 \%$ after 6 months. In

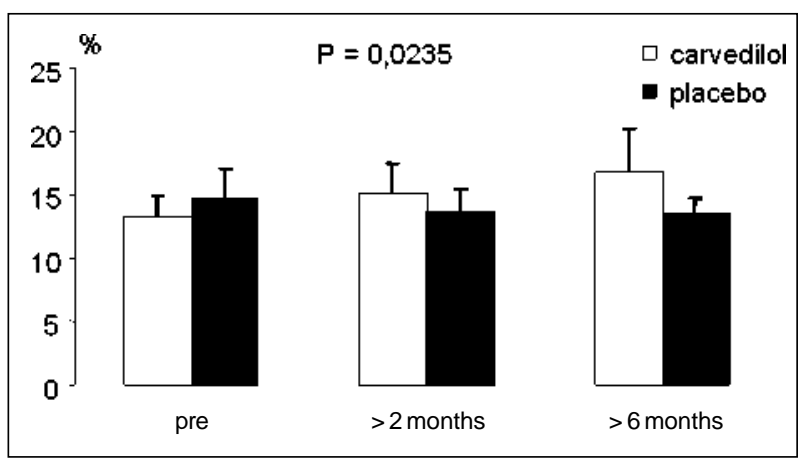

Fig. 6-Comparison of the evolution in left ventricular shortening fraction on echocardiogram between the groups using carvedilol or placebo in the pretreatment phase (pre), after 2 months of medication use (>2 months), and after 6 months of medication use ( $>6$ months).

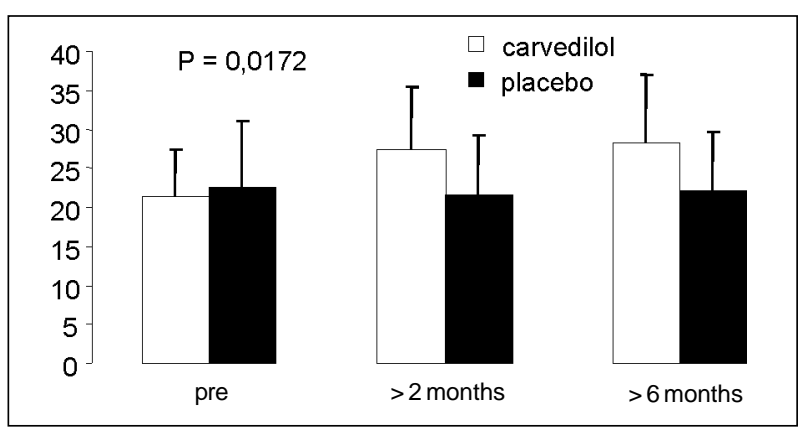

Fig. 7 - Comparison of the evolution in left ventricular ejection fraction on radionuclide ventriculography between the groups using carvedilol or placebo in the pretreatment phase (pre), after 2 months of medication use ( $>2$ months), and after 6 months of medication use (>6 months). 


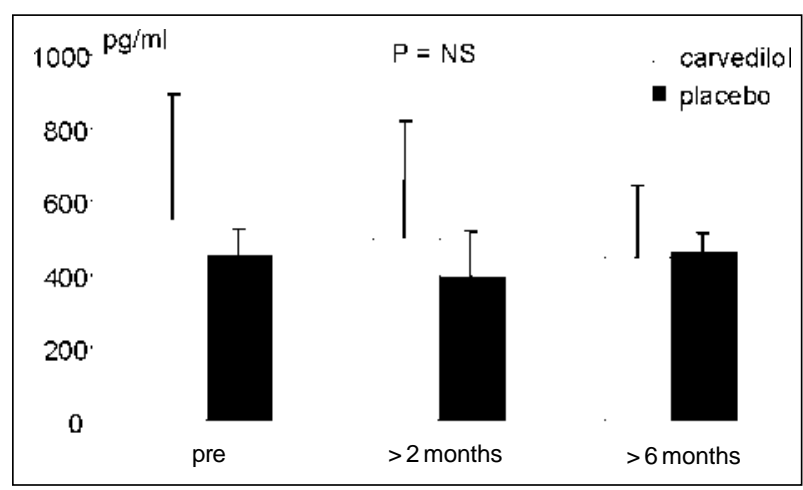

Fig. 8 - Comparison of the evolution in serum concentration of nora-drenaline between the groups using carvedilol or placebo in the pretreatment phase (pre), after 2 months of medication use ( $>2$ months), and after 6 months of medication use ( $>6$ months).

the group using placebo, the mean remained unaltered, around $22 \%(\mathrm{p}=0.0172)$.

In regard to the serum level of noradrenaline (fig. 8), no significant difference between the means of the groups occurred during the study.

\section{Discussion}

Our results show that addition of carvedilol to the treatment of patients with heart failure reduces heart rate and improves cardiac function. Despite improvement in cardiac function, however, in the patients selected, we did not notice significant changes in the left ventricular diameters or in serum levels of noradrenaline.

Explanation of the mechanisms of action of carvedilol remains speculative. It is known that carvedilol promotes reduction in cardiovascular mortality, in hospital stay, and in the need to readjust medication ${ }^{14}$ due to a probably antioxidant, antiproliferating ${ }^{11}$, and antiarrhythmic ${ }^{15}$ beta- and alpha-1-blocking action.

Our study did not show any clinical worsening in any of the groups. In a study of 415 individuals ${ }^{16}$, the patients using carvedilol with heart failure caused by ischemia with an ejection fraction lower than $45 \%$ showed a tendency toward worsening functional class compared with the placebo group. Two thirds of the patients, however, maintained their functional classes unaltered during the 6 months of assessment. Our study showed that almost $70 \%$ of the patients using placebo remained clinically unchanged during the whole evaluation; in the group using carvedilol, however, $73 \%$ of the patients improved their functional classes. Other double-blind randomized studies ${ }^{17-19}$ have shown that, despite the ischemic or nonischemic cause, the patients have a significant improvement in symptoms and functional class with the use of carvedilol as compared with the placebo group. Nevertheless, so far the addition of carvedilol to the treatment of heart failure has not shown significant alterations in the quality of life of the patient with heart failure in the total, physical, and emotional dimensions ${ }^{20}$.

In our study, addition of carvedilol to the conventional
Carvedilol in heart failure due to dilated cardiomyopathy

therapy for heart failure was well tolerated by the patients, even in doses higher than those recommended. The rate of exclusion from the study due to intolerance to the drug was equal in both groups, and only 2 patients on carvedilol did not tolerate a dose higher than $50 \mathrm{mg} /$ day. Up to now, a dose higher than $50 \mathrm{mg} /$ day has been recommended only for patients weighing more than $75 \mathrm{~kg}^{21}$. In our study, the mean weight of the patients ranged from $65 \mathrm{~kg}$ to $70 \mathrm{~kg}$. This result allows us to increase the dose of the beta-blocker within the already tested limits, until the best desired effect is obtained.

The 26\% reduction in the heart rate observed in the group using carvedilol shows a clear beta-blocking effect. Other studies have also shown a reduction in the heart rate with the use of carvedilol, which ranged from $13 \%$ to $30 \%$ in the pretreatment phase ${ }^{16,18,22}$. The use of a second generation beta-blocker, such as metoprolol, in patients with heart failure, causes a similar reduction in heart rate, even though carvedilol, a third generation beta-blocker, causes additional hemodynamic effects ${ }^{23,24}$ similar to the vasodilators without reflex tachycardia ${ }^{25}$.

Reduction in left ventricular diameters with the use of carvedilol is controversial and may be related to the size of the sample or severity of the disease studied. A study ${ }^{17}$ of 49 patients with diverse causes did not show an alteration in left ventricular diameters with the addition of carvedilol to the conventional therapy for heart failure. Another Brazilian study ${ }^{22}$ with a small sample of 21 patients refractory to the treatment of heart failure of diverse causes has shown a significant reduction in left ventricular diastolic diameter with the use of carvedilol. A study ${ }^{16}$ of 415 patients with heart failure due to ischemia has shown a significant reduction in ventricular diameters after 6 months of carvedilol use. We may not have found a significant reduction in left ventricular diameters because of the size of the sample studied.

In spite of the ventricular diameters, a number of studies ${ }^{16,18,20}$ have shown an improvement in ventricular function in patients with heart failure treated with carvedilol, as well as hemodynamic improvement, a reduction in peripheral resistance ${ }^{15}$, an improvement in pulmonary capillary wedge pressure ${ }^{23}$, in pulmonary pressure and in systolic volume ${ }^{16}$.

Our patients showed an improvement in left ventricular function with a significant improvement in shortening fraction in the myocardial fiber on echocardiogram and improvement in ejection fraction on radionuclide ventriculography, with the association of carvedilol with the conventional therapy of heart failure. Some studies show similar improvement in ventricular performance with the use of carvedilol ${ }^{17}$, even when compared with the group using captopril $^{21}$ or even another beta-blocker, metoprolol ${ }^{22}$. Improvement in ejection fraction is also directly related to the dose of carvedilol, being more marked in those patients with a nonischemic cause ${ }^{19}$.

Even though we have not observed any significant difference in the serum level of noradrenaline in samples of peripheral blood between the groups using carvedilol or placebo, another study ${ }^{22}$ has found a reduction in the concentration of noradrenaline in the coronary sinus in the 
group using carvedilol as compared with the group using metoprolol. Our results show a tendency toward reduction in the level of serum noradrenaline in a sample of peripheral blood with the use of carvedilol; we believe, however, that the small number of patients and the proportion of standard deviation have not allowed statistically significant results.

Therapeutical agents may interfere with myocardial remodeling and reduce the progression of ventricular dysfunction, if they interfere with myocyte hypertrophy and apoptosis secondary to stress, to the action of angiotensin and of the adrenergic stimulus ${ }^{26}$. Showing that carvedilol reduces heart rate and improves left ventricular function, we may infer that carvedilol works on the process of cardiac remodeling, leading to a better adaptation of the myocardial dysfunction. Briefly, the use of a beta-blocker in heart failure prevents and reverses myocardial dysfunction and remodeling mediated by an adrenergic stimulus ${ }^{27}$. It is believed that the beta-blocker improves cardiac function in patients with chronic heart failure with progressive up-regulation in the depression of myocardial beta-receptors ${ }^{28}$.

As limitations of the study, we can cite the small sample of patients and the high rate of exclusion. Our study did not compare the need for reduction in medications, as carvedilol was used in association with other medications. Further studies should assess this need for reducing the medication aiming to avoid polypharmacy and its complications in the treatment of heart failure.

In conclusion, use of carvedilol in doses higher than those recommended associated with the conventional therapy for heart failure in patients with idiopathic dilated cardiomyopathy has resulted in improvement in cardiac function, in spite of the unchanged ventricular diameters, possibly due to interference with the adaptive mechanisms of cardiac remodeling. The tolerance to the drug evidenced allowed us to increase the dose of carvedilol until the desired beta-blocking effect was obtained.

\section{References}

1. Cohn JN. Overview of the treatment of heart failure. Am J Cardiol 1997; 80(11A): $2 \mathrm{~L}-6 \mathrm{~L}$.

2. The CONSENSUS Trial Study Group. Effects of Enalapril on Mortality in Severe Congestive Heart Failure: Results of the Cooperative North Scandinavian Enalapril Survival Study (CONSENSUS). N Engl J Med 1987; 316: 1429-35.

3. The SOLVD investigators. Effect of Enalapril on Mortality and Survival in Patients With Reduced Left Ventricular Ejection Fractions and Congestive Heart Failure. N Engl J Med 1991; 325: 293-302.

4. Cohn JN, Johnson G, Ziesche S, et al. A comparison of enalapril with hydralazine-isosorbide dinitrate in the treatment of chronic congestive heart failure. $\mathrm{N}$ Engl J Med 1991; 325: 303-10.

5. Fowler MB. Controlled trials with beta blockers in heart failure: metoprolol as the prototype. Am J Cardiol 1993; 45C-53C

6. Packer M, Bristow MR, Cohn JN, et al. For The US carvedilol heart failure study group. The effect of carvedilol on morbidity and mortality in patients with chronic heart failure. N Engl J Med 1996; 334: 1349-55.

7. Feuerstein GZ, Bril A, Ruffolo Jr RR. Protective effects of carvedilol in the myocardium. Am J Cardiol 1997; 80(11A): 41L-45L.

8. Feuerstein GZ, Ruffolo RR. Carvedilol, a novel multiple action antihypertensive agent with antioxidant activity and vascular protection. Eur Heart J 1995; 16(suppl. F): 38-42.

9. Freitas HFG, Nastari L, Mansur AJ, et al. Dinâmica da avaliação de pacientes para transplante cardíaco ou cardiomioplastia. Arq Bras Cardiol 1994; 62: 233-7.

10. Andersson B, Caidahl K, Waagstein F. Idiopathic dilated cardiomyopathy among Swedish patients with congestive heart failure. Eur Heart J 1995, 16: 53-60.

11. Cleland JGF, Swedberg K. Carvedilol for heart failure, with care. Lancet 1996; 347: 1199-1201.

12. Bristow MR, Gilbert EM, Abraham WT, et al. For The MOCHA Investigators Carvedilol produces dose-related improvements in left ventricular function and survival in subjects with chronic heart failure. Circulation 1996; 94: 2807-16.

13. Winer BJ, Brown DR and Michels. Statistical Principles in Experimental Design. New York: MacGraw-Hill, 1991.

14. Chatterjee K. Heart failure therapy in evolution. Circulation 1996; 94: 2689-93.

15. Wright DJ, Cooke GA, Tan LB. Intractable recurrent ventricular tachycardia in dilated cardiomyopathy controlled by a vasodilating $\beta$-blocker. Heart 1997; 77 : $581-2$.
16. Australia-New Zealand Heart Failure Research Collaborative Group. Effects of carvedilol, a vasodilator $\beta$-blocker, in patients with congestive heart failure due ischemic heart disease. Circulation 1995; 92: 212-8.

17. Krum H, Sackner-Bernstein JD, Goldsmith RL, et al. Double-blind, placebocontroled study of the long-term efficacy of carvedilol in patients with severe chronic heart failure. Circulation 1995; 92: 1499-1506.

18. Metra M,Nardi M, Giubbini R, Cas LD. Effects of short and long-term carvedilol administration on rest and exercise hemodynamic variables, exercise capacity and clinical conditions in patients with dilated idiopathic dilated cardiomyopathy. J Am Coll Cardiol 1994; 24: 1678-87.

19. Packer M, Colucci WS, Sackner-Bernstein JD, et al. PRECISE Study Group. Double-blind, placebo-controlled study of the effects of carvedilol in patients with moderate to severe heart failure-The PRECISE Trial. Circulation 1996; 94: 2793-9.

20. Colucci WS, Packer M, Bristow MR, et al. For The US Carvedilol heart failure tudy group. Carvedilol inhibits clinical progression in patients with mild symptoms of heart failure. Circulation 1996; 94: 2800-6.

21. Olsen SL, GilbertEM, Renlund DG, Taylor DO, YanowitzFD, Bristow MR. Carvedilol improves left ventricular function and symptoms in chronic heart failure: a double-blind randomized study. J Am Coll Cardiol 1995; 25: 1225-31.

22. BocchiEA, BacalF, Bellotti G, Carrara D, Ramires JAF. Efeitos do carvedilol (bloqueador $\beta_{1} \beta_{2} \alpha_{1}$ ) na insuficiência cardíaca refratária. Arq Bras Cardiol 1998; 71: 169-73.

23. Jansson K, Karlberg E, Nylander E, Karisson E, Nyquist O, Dahiström U. More favorable haemodynamic effects from metoprolol than from captopril in patients with dilated cardiomyopathy. Eur Heart J 1997; 18: 1115-21.

24. Gilbert EM, Abraham WT, Olsen S, et al. Comparative hemodinamic, left ventricular functional, and antiadrenergic effects of chronic treatment with metoprolol versus carvedilol in the failing heart. Circulation 1996; 94: 2817-25.

25. DasGupta $P$, Broadhurst $P$, Lahiri A. The effects of intravenous carvedilol, a new multiple action vasodilatory $\beta$-blocker, in congestive heart failure. J Cardiovasc Pharmacol 1991; 18(suppl.4): S12-S16.

26. Colucci WS. Molecular and cellular mechanisms of myocardial failure. Am J Cardiol 1997; 80(11A): 15L-25L.

27. Bristow MR. Mechanism of action of beta-blocking agents in heart failure. Am J Cardiol 1997; 80(11A): 26L-40L.

28. Waagstein F. Beta-blockers in heart failure. Cardiology 1993; 82(suppl. 3): 13-8. 